\title{
PREPARATION AND ANTIBACTERIAL ACTIVITY OF CMC-g-P (SPMA)/SILVER NANOCOMPOSITE HYDROGEL
}

\author{
AHMED SALAMA, MAHA A. YOUSSEF, ${ }^{*}$ ABEER A. HASSAN ${ }^{* *}$ and NASSER S. AWWAD ${ }^{* * *}$ \\ Cellulose and Paper Department, National Research Centre, 33 El-Bohouth Str., \\ Dokki, P.O. 12622, Giza, Egypt \\ "Analytical Chemistry and Control Department, Hot Laboratories Center, Atomic Energy Authority of \\ Egypt, P.O. 13759 Abu Zaabal, Cairo, Egypt \\ ** Chemistry Department, Faculty of Science for Girls, Ain Shams University, Cairo, Egypt \\ ${ }^{* * *}$ Department of Chemistry, Faculty of Science, King Khalid University, \\ P.O. Box 9004 Abha, Saudi Arabia \\ \Corresponding author: Ahmed Salama,Ahmed_nigm78@yahoo.com
}

Received December 5, 2018

A novel hydrogel was synthesized by grafting of poly(3-sulfopropyl methacrylate) (PSPMA) onto carboxymethyl cellulose (CMC) via the free radical polymerization technique, using $\mathrm{N}, \mathrm{N}^{\prime}$-methylene bisacrylamide (MBA) as crosslinker and potassium persulphate (KPS) as initiator. CMC-g-P(SPMA) hydrogel and glucose were used as stabilizing and reducing agents, respectively, for the preparation of silver nanoparticles loaded hydrogel by a facile and green technique. The synthesized CMC-g- P(SPMA)/Ag nanocomposite was characterized by FTIR, UV-Vis spectroscopy, XRD, TGA, TEM and SEM techniques. The formed silver nanoparticles exhibited spherical shape and were homogenously dispersed in the hydrogel. These particles had an average particle size of $18 \mathrm{~nm}$, as calculated from TEM results. The antibacterial and antifungal performance of the CMC-g-P(SPMA)/Ag nanocomposite was investigated and the results recommended the nanocomposite as an efficient and eco-friendly antibacterial and antifungal material.

Keywords: carboxymethyl cellulose, hydrogel, silver nanoparticles, nanocomposite

\section{INTRODUCTION}

In the trend towards environmentally friendly and more sustainable chemistry, polysaccharides have attracted a great deal of attention as a potential substitute for non-biodegradable and synthetic polymers. ${ }^{1,2}$ Moreover, polysaccharides have also a lot of other advantages, for instance, their low cost, non-toxicity, biodegradability and their virtually unlimited availability. ${ }^{3,4}$ However, the applications of polysaccharides are hindered in advanced fields, such as biomaterials, due to their poor functionality, mechanical and antibacterial properties. Recently, polysaccharides/inorganic hybrids were developed as new active packaging materials for controlling microbial growth in food products. ${ }^{5-7}$ Moreover, polysaccharides/calcium phosphate hybrids were applied for bone tissue engineering, which require bioactivity and biocompatibility of the used scaffolds. ${ }^{1}$ Cellulose has become an alternative non-toxic and bioactive polymer for preparing novel hybrid materials. Carboxymethyl cellulose (CMC) has been applied for the design of novel engineered hydrogels for biomedical, environmental and industrial applications. ${ }^{8,9}$ In our previous study, a CMC-g-P(DMEMA) hydrogel was prepared and demonstrated to be an efficient anionic dye adsorbent from waste water. $^{10}$ Functionalization of cellulose by incorporating nanoparticles is a promising approach for developing various hybrid systems. Olatunji et al. ${ }^{11}$ designed a new composite material based on regenerated nanocellulose, $\mathrm{TiO}_{2}$ and $\mathrm{ZnO}_{2}$. The prepared nanocomposite was strongly bound, possessed strong hydrophobic nature and a great potential for delivering tramadol hydrochloride. Among noble metal nanoparticles, silver nanoparticles have been applied in biomaterials due to their excellent antibacterial properties and non-toxicity. ${ }^{12}$ Silver nanocomposite hydrogels, combining the properties of an organic hydrogel and inorganic nanostructured materials, have recently gained 
significant attention. ${ }^{13}$ The synergetic effects between the organic polymer and silver nanoparticles are responsible for introducing new properties to the formed nanocomposite. Compared to classic hydrogels, nanocomposites have been introduced in numerous applications, such as catalysis, ${ }^{14}$ design of sensors ${ }^{15}$ and biotechnology. ${ }^{16}$ Silver nanocomposites, which possess antibacterial activity, have been applied in many cosmetic and biomedical materials. However, silver nanoparticles tend to minimize their surface energy by forming aggregations during their preparation. It is critical to find appropriate and efficient support materials to load silver nanoparticles to avoid this shortcoming. Wei Shao et al. prepared silver loaded graphene oxide nanoparticles as an antibacterial nanocomposite. The authors used glucose and starch to reduce and stabilize silver ions on the surface of graphene oxide nanosheets. The results showed that uniform and compactly deposited silver nanoparticles, of approximately $22 \mathrm{~nm}$, were formed. Moreover, the graphene oxide/silver nanocomposite displayed low cytotoxicity and effective antibacterial activity. ${ }^{17}$

Few promising studies were carried out to investigate polymer containing sulfonic groups for industrial applications. For example, Tugce Turhan et al. prepared a composite catalyst system from PSPMA hydrogel loaded with Co and $\mathrm{Ni}$, which was further applied for the hydrolysis of $\mathrm{NaBH}_{4}$ for hydrogen production. ${ }^{18}$ Moreover, sulfonated polymers have recently been applied as adsorbents for waste water treatment. Few articles have been reported on the grafting of monomers containing sulfonyl groups onto cellulose via the graft polymerization technique. ${ }^{19}$

The current study aims to use a facile and sustainable method for preparing a silver nanocomposite. A CMC-g-P (SPMA) hydrogel was prepared and investigated as a supporting hydrogel and stabilizer during the reduction of silver ions to silver nanoparticles. The nanocomposite, CMC-g-P(SPMA)/Ag, was investigated as a sustainable and antibacterial material.

\section{EXPERIMENTAL \\ Materials}

Carboxymethyl cellulose sodium salt ( $>99.5 \%)$ was purchased from Fluka Biochemika. 3-Sulfopropyl

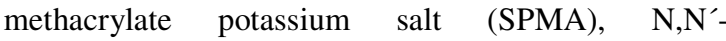
methylenebisacrylamide (MBA) and potassium persulphate (KPS) were purchased from SigmaAldrich. Silver nitrate was purchased from Alpha Chemika. Other chemicals were of analytical grade and used as received without further purification.

\section{Preparation of CMC-g-PSPMA/Ag nanocomposite}

The CMC-g-P (SPMA) hydrogel was synthesized by adding $2 \mathrm{~g} \mathrm{CMC}$ to $100 \mathrm{~mL}$ double-distilled water in a three-neck reactor to prepare a $2 \%(\mathrm{w} / \mathrm{v})$ solution. The solution was stirred in a thermostated water bath adjusted to $70{ }^{\circ} \mathrm{C}$ and purged with nitrogen for 30 minutes. The initiator $(0.017 \mathrm{~mol} / \mathrm{L})$ was added, followed by the addition of SPMA monomer (0.4 $\mathrm{mol} / \mathrm{L})$, then the crosslinker $(0.007 \mathrm{~mol} / \mathrm{L})$ and the reaction mixture was stirred until complete gelation. The gel product was chopped to small pieces and allowed to completely swell in excess amounts of double-distilled water several times along three days to remove the unreacted molecules.

The CMC-g-PSPMA/Ag nanocomposite was prepared by stirring $2 \mathrm{~g}$ hydrogel immersed in $50 \mathrm{~mL}$ distilled water with $0.1 \mathrm{~g}$ silver nitrate treated by ultrasonication for 10 minutes. Then, $0.2 \mathrm{~g}$ of glucose dissolved in $2 \mathrm{~mL}$ distilled water was added, followed by heating for $4 \mathrm{~h}$ at $80{ }^{\circ} \mathrm{C}$. The color of the reaction mixture turned from dark brown to gray and finally to dark green. The synthesized CMC-g-P(SPMA)/Ag nanocomposite was separated and washed three times using deionized water, and then was freeze-dried using liquid nitrogen.

\section{Swelling properties}

The equilibrium swelling\% of the synthesized CMC-g-P(SPMA) and the nanocomposite hydrogel was measured in different solutions: distilled water, acidic, basic and $\mathrm{NaCl}$ solutions (0.3, 0.6 and 0.9 wt $\%)$. The swelling percent was calculated according to Equation 1:

Swelling $\%=\left[\left(W_{t}-W_{o}\right) / W_{o}\right] \times 100$

where $W_{\mathrm{o}}$ is the initial weight and $W_{\mathrm{t}}$ the weight of the samples at time $t^{20}$

\section{Characterization}

Fourier transform infrared spectroscopy (FT-IR) was performed on a FTIR (Mattson 5000 FTIR spectrometer), using $\mathrm{KBr}$ discs, in the range of 4000$500 \mathrm{~cm}^{-1}$. UV-visible absorption spectra were recorded on a JASCO V-650 spectrophotometer in the range of 200-700 nm. Scanning electron microscopy was carried out on a Quanta 250 FEG (Field Emission Gun), provided with an EDX Unit (Energy Dispersive X-ray Analyses), with accelerating voltage of $30 \mathrm{~K}$. Transmission electron microscopy (TEM) images were taken with a JEOL JEM-2100 electron microscope at $100 \mathrm{k} \times$ magnification, with an acceleration voltage of $120 \mathrm{kV}$. XRD was performed using an X-ray diffractometer (PANalytical, Netherlands), with a monochromatic $\mathrm{CuK}_{\alpha}$ radiation source $(\lambda=0.154 \mathrm{~nm})$ 
in the step-scan mode. Thermogravimetric analysis was done on a PerkinElmer TGA7 thermogravimetric analyzer under nitrogen.

\section{Antibacterial test}

The antibacterial activity of the CMC-g-P (SPMA) hydrogel and the nanocomposite was evaluated using the agar disk diffusion method against Escherichia coli (E. coli), Staphylococcus aureus (S. aureus), Candida albicans (C. albicans) and Aspergillus flavus (A. flavus). $15 \mu \mathrm{L}$ from the aqueous dispersion of the examined samples was added onto $10 \mathrm{~mm}$ filter paper, dried and sterilized by an ultraviolet lamp for 60 minutes. The examined films were placed on the seeded agar plate. After 24 and $72 \mathrm{~h}$ of incubation at $37{ }^{\circ} \mathrm{C}$, the diameters of the inhibition zones were measured.

\section{RESULTS AND DISCUSSION}

Scheme 1 shows the proposed steps for the preparation of the CMC-g-P(SPMA) hydrogel via the free radical polymerization technique. The prepared hydrogel, which contains different acid functional groups, sulfonic and carboxylic groups, is expected to adsorb and stabilize the formed silver nanoparticles. The CMC-g-P(SPMA) hydrogel was prepared with SPMA as monomer, MBA as cross-linker and KPS as initiator, using the procedures described in our previous study. ${ }^{10}$

\section{Characterization of the hydrogel and nanocomposite}

The graft co-polymerization was proved by FT-IR spectroscopy, as shown in Figure 1A. CMC shows characteristic bands at 1631, 2925, and $3457 \mathrm{~cm}^{-1}$, which are assigned to the carboxyl group, asymmetric $\mathrm{C}-\mathrm{H}$ stretching and $\mathrm{O}-\mathrm{H}$ stretching vibration, respectively. ${ }^{21}$ However, the CMC-g-P(SPMA) hydrogel shows new characteristic peaks at 1086 and $626 \mathrm{~cm}^{-1}$, which are attributed to the stretching vibrations of $\mathrm{S}=\mathrm{O}$ and $\mathrm{S}-\mathrm{O}$, respectively. ${ }^{22}$ Moreover, an additional new band at $1718 \mathrm{~cm}^{-1}$ for the CMC-g$\mathrm{P}$ (SPMA) hydrogel was assigned to the carbonyl $(\mathrm{C}=\mathrm{O})$ groups in the $\mathrm{P}(\mathrm{SPMA})$ chains. The increased intensity of the absorption peak at 3469 $\mathrm{cm}^{-1}$ may refer to the water bonded to the sulfonic groups. $^{23}$

UV-visible spectroscopy was used to monitor the formation of silver nanoparticles on the CMCg-P (SPMA) hydrogel. A weak characteristic peak at $292 \mathrm{~nm}$ was observed in the UV-visible spectrum of the CMC-g-P(SPMA) hydrogel, which is ascribed to the $n-\pi^{*}$ transitions of the $\mathrm{C}=\mathrm{O}$ bonds. As shown in the UV-visible spectra (Fig. 1B), the formation of silver nanoparticles is indicated by the surface plasmon resonance peak at around $400 \mathrm{~nm}$ in the CMC-g-P(SPMA)/silver nanocomposite, which is similar to previous findings. ${ }^{17}$ Figure $1 \mathrm{C}$ exhibits a cross-section image of the CMC-g-P (SPMA) hydrogel obtained by SEM. The morphology of CMC-g-P (SPMA) exhibits a well-defined porous and interconnected three-dimensional framework structure. The homogeneously distributed porous structure of the hydrogel allows easy access of aqueous solutions inside the network structure.

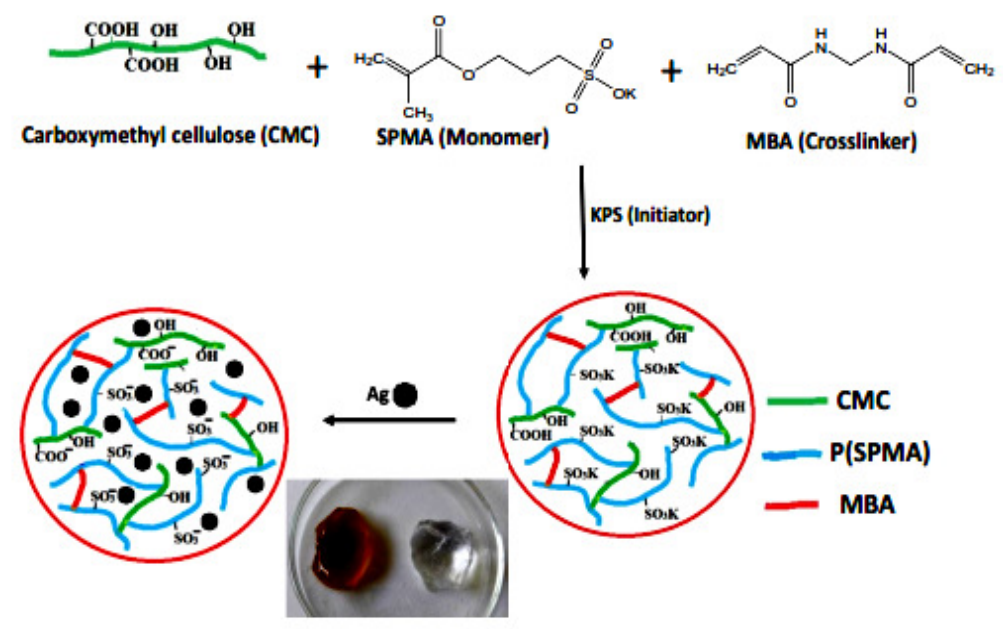

Scheme 1: Proposed mechanism for the preparation of CMC-g-P (SPMA)/Ag nanocomposite 

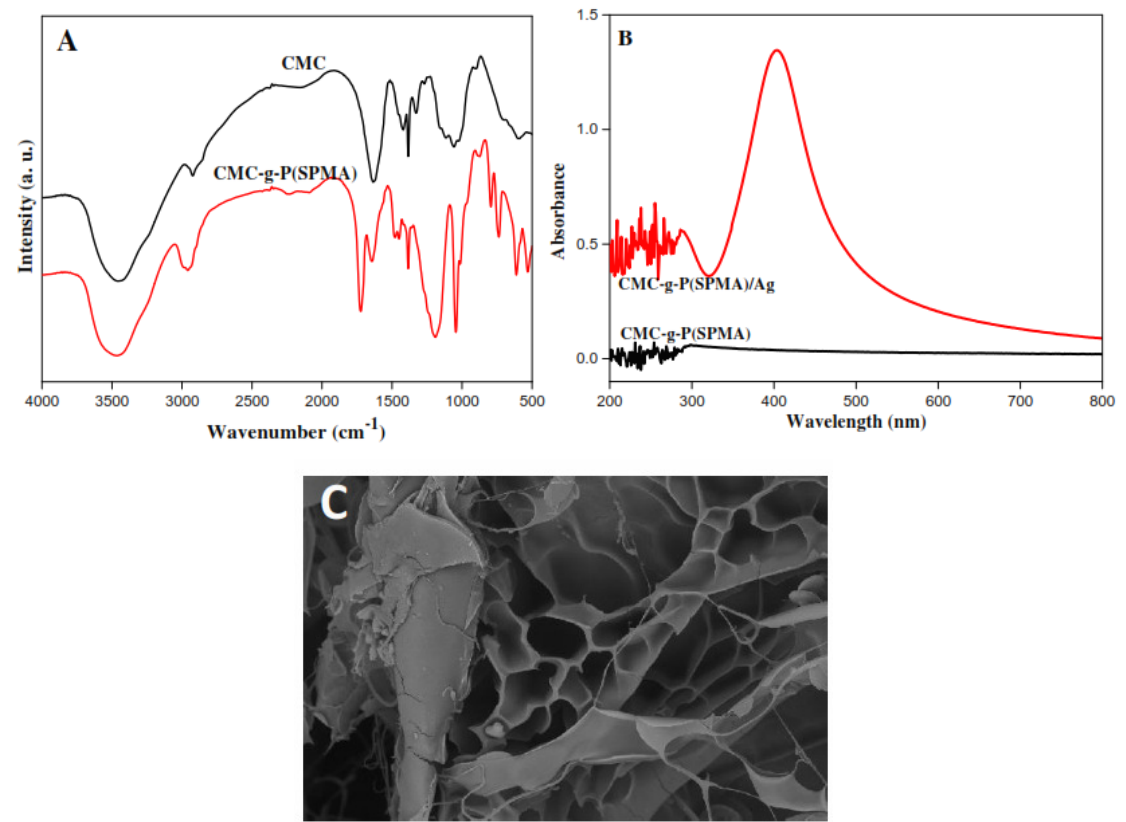

Figure 1: FT-IR spectra of CMC and CMC-g-P(SPMA) hydrogel (A), UV-vis spectroscopy of CMC-g-PSPMA hydrogel and CMC-g-P(SPMA)/Ag nanocomposite (B) and SEM of CMC-g-P(SPMA) hydrogel (C)
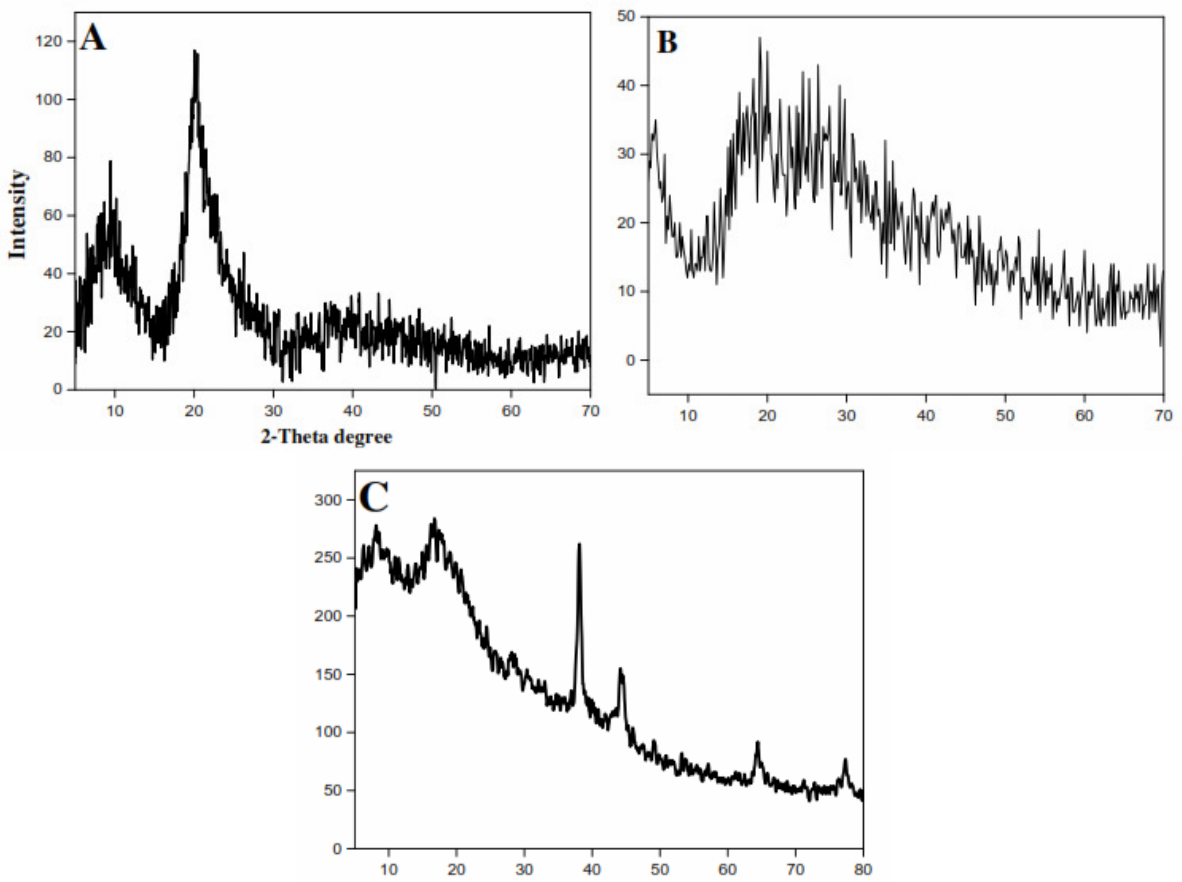

Figure 2: XRD of CMC (A), CMC-g-P(SPMA) hydrogel (B) and CMC-g-P(SPMA)/Ag nanocomposite

$\mathrm{X}$-ray diffraction (XRD) is an efficient tool to investigate the degree of crystallinity of polymeric materials and to prove the hydrogel formation. XRD patterns of CMC and CMC-g$\mathrm{P}(\mathrm{SPMA})$ hydrogel are presented in Figure 2. CMC shows sharp reflections at $2 \theta 9.4$ and $20.1^{\circ}$, which are attributed to the partially crystalline structure of the neat CMC. The crystalline structure of CMC originates from strong interand intramolecular hydrogen bonds between the CMC chains. ${ }^{22}$ However, these two bands became very marked and broad in the CMC-g-P(SPMA) hydrogel, indicating its amorphous glassy structure. These variations suggest that graft 
copolymerization destroys the intermolecular hydrogen bonds between oxygen containing functional groups in the CMC chains. XRD results proved that the SPMA monomer was grafted onto the $\mathrm{CMC}$ chains to form the CMC-gP(SPMA) hydrogel and the grafting process destroys the crystal structure of CMC. The crystalline nature of the CMC-g-P(SPMA)/Ag nanocomposite was established by $\mathrm{X}$-ray crystallography, as shown in Figure 2C. The diffracted intensities at 38.1, 44.5, 64.4, and 77.6 can indexed to the (111), (200), (220) and (311) planes of the face-centered cubic crystal structure of silver. ${ }^{24}$

The microstructure of the CMC-gP(SPMA)/Ag nanocomposite showed a smooth surface with homogenously distributed wrinkles. Under high magnification, randomly distributed cracks can be observed. Figure 3 (D, E, F and G) shows the X-ray elemental maps of carbon, oxygen, sulfur and silver detected by energy dispersive X-ray spectroscopy. The maps indicate fairly homogeneous elemental distributions on the length of a few micrometers, suggesting uniform distribution of the silver nanoparticles over the micrometer length scale. EDX analysis also confirms the formation the CMC-g-P (SPMA)/Ag nanocomposite, as shown in Figure $3 \mathrm{H}$.

Transmission electron microscopy (TEM) was used to analyze the morphological aspects of the CMC-g-P(SPMA)/Ag nanocomposite. Figure 4A reveals the formation of silver nanoparticles, represented by dark spots, through an almosttransparent CMC-g-P(SPMA) hydrogel. Moreover, the TEM image shows that the silver nanoparticles (black dots) are spherical and uniformly dispersed inside the network of the CMC-g-P(SPMA) hydrogel. The size distribution of the deposited silver nanoparticles is relatively narrow, with a mean diameter of about $18 \mathrm{~nm}$. These results exhibit that the CMC-g-P(SPMA) hydrogel chains play a critical role in the process of nucleation and stabilization of he formed silver nanoparticles, preventing their agglomeration. A HRTEM image shows that the silver nanoparticles displayed a multitwinned structure. In addition, the measured fringe lattice of the silver nanoparticles is $0.24 \mathrm{~nm}$, attributed to the (111) plane.
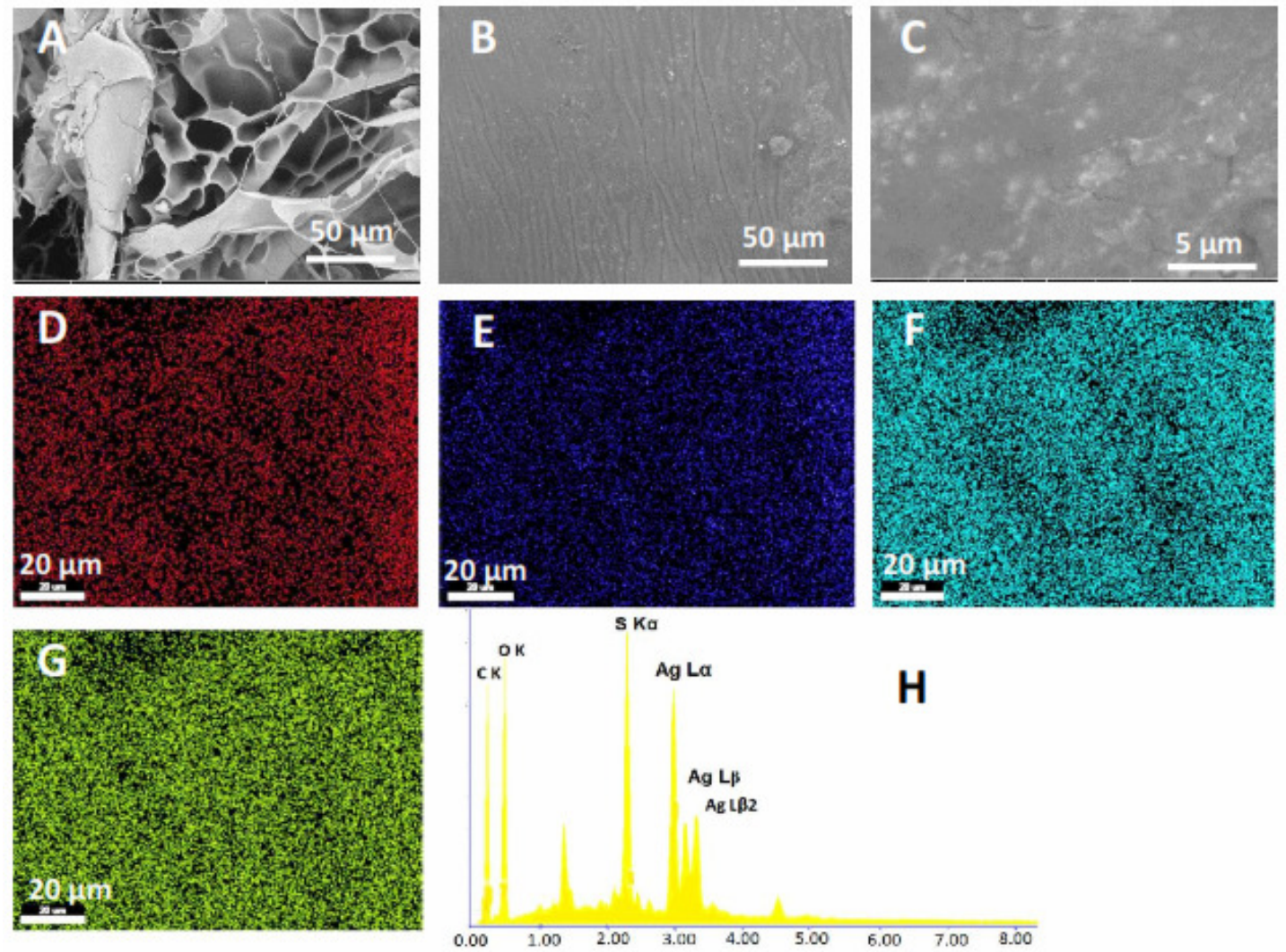

Figure 3: SEM images of CMC-g-P(SPMA) hydrogel (A), CMC-g-P (SPMA)/Ag nanocomposite at different magnification (B, C), maps of carbon (D), oxygen (E), sulfur $(F)$, silver $(G)$ and EDX of

CMC-g-P (SPMA)/Ag nanocomposite $(\mathrm{H})$ 

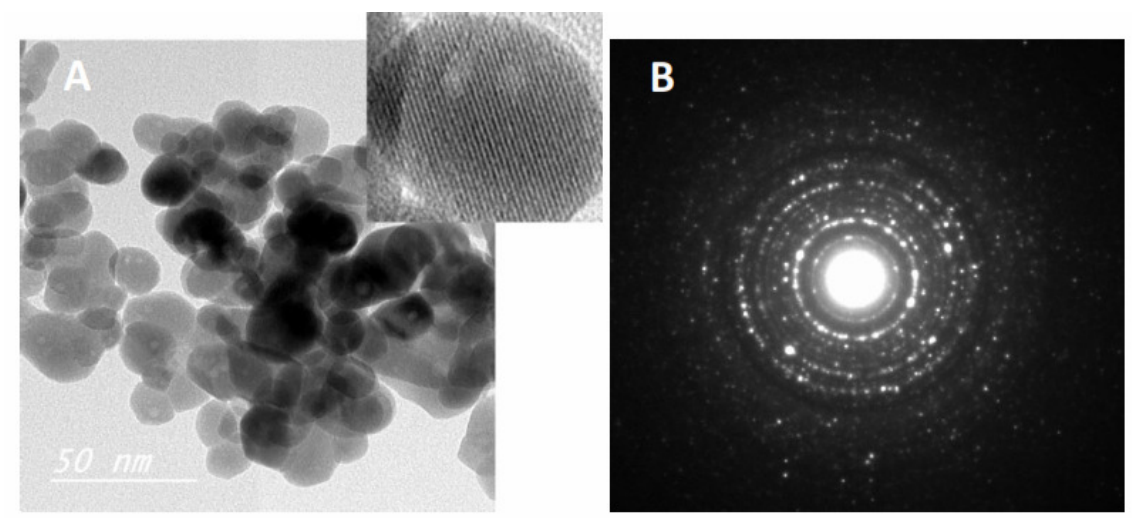

Figure 4: TEM spectroscopy, HRTEM (A) and SAED pattern (B) of CMC-g-P(SPMA)/Ag nanocomposite

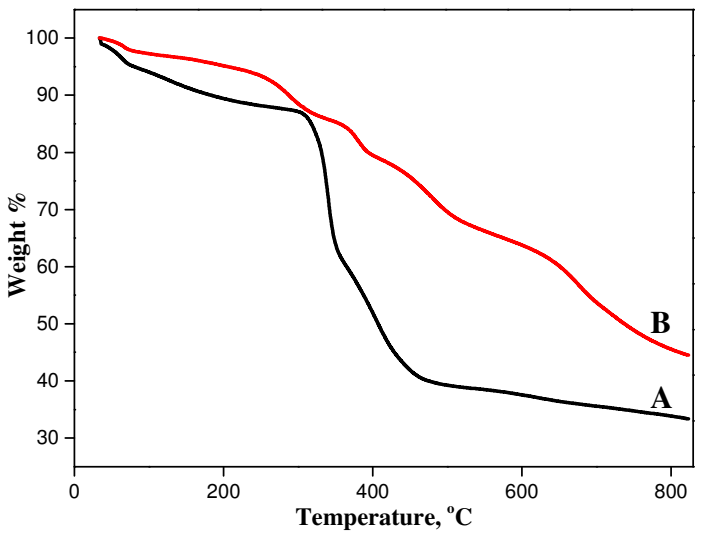

Figure 5: TGA curves of CMC-g-P(SPMA) hydrogel (A) and CMC-g-P (SPMA)/Ag nanocomposite (B)

The crystallinity of the silver nanoparticles was further evaluated by selected-area electron diffraction (SAED). The SAED pattern with bright circular rings proved the crystalline nature of the silver nanoparticles. The strongest pattern (inner ring) shows the characteristic diffraction rings, which are likely attributed to the (111) plane (Fig. 4B). These results suggest the role of carboxylic and sulfonic groups in the hydrogel in stabilizing the face-centered cubic crystal structure of silver.

TGA is used to display the compositional analysis and thermal stability with respect to changes in mass as a function of time and temperature. The TGA thermograms for the two samples are completely different, as illustrated in Figure 5. The CMC-g-P(SPMA) hydrogel shows two main regions of mass loss. The initial weight loss (9.3\%), observed below $200{ }^{\circ} \mathrm{C}$, can be attributed to the evaporation of physically adsorbed water. The second sharp step, observed

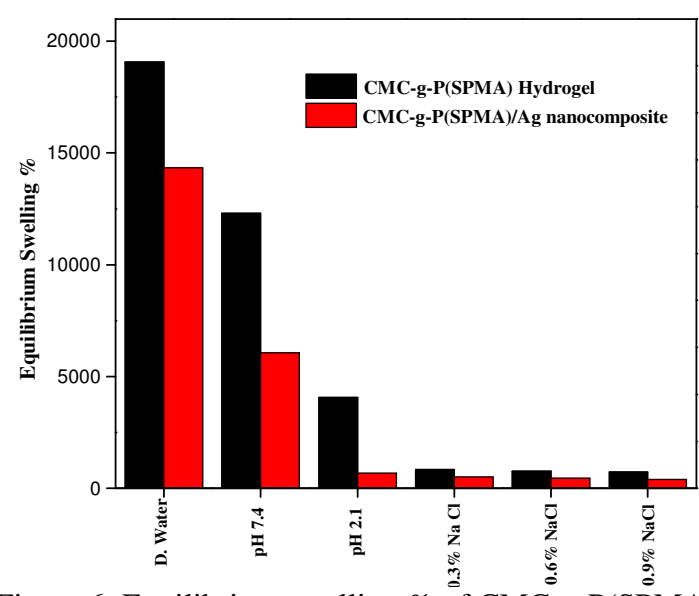

Figure 6: Equilibrium swelling \% of CMC-g-P(SPMA) hydrogel and CMC-g-P(SPMA)/Ag nanocomposite in different media

between 300 and $470{ }^{\circ} \mathrm{C}$, indicates the decomposition of the CMC-g-P(SPMA) hydrogel. However, the CMC-g-P(SPMA)/Ag nanocomposite was gradually decomposed and its weight loss reached $28.1 \%$ at $480{ }^{\circ} \mathrm{C}$. In addition, the residual mass of the CMC-g-P(SPMA) hydrogel and of the CMC-g-P(SPMA)/Ag nanocomposite at $800{ }^{\circ} \mathrm{C}$ is 33.9 and 45.5 , respectively. TGA results showed that the CMCg-P(SPMA)/Ag nanocomposite has higher thermal stability, compared with the CMC-gP(SPMA) hydrogel.

\section{Swelling properties of the hydrogel and nanocomposite}

The swelling capacity of the CMC-gP(SPMA) hydrogel and of the nanocomposite would be decisive for their later use in biomaterials. The hydrogel and the nanocomposite were soaked in different media: water, acidic solution, basic solution and saline 
solution. The equilibrium swelling ratios as a function of the swelling medium are shown in Figure 6. The equilibrium swelling of the nanocomposite in all the solvents was lower than that of the hydrogel, indicating that the silver nanoparticles played the expected role in crosslinking the hydrogel network. The highest swelling ratios were observed in water and in the weak alkaline solution $(\mathrm{pH} 7.4)$, while in acidic medium and in physiological solutions $(3 \%, 6 \%$ and $9 \% \mathrm{NaCl}$ ), the samples displayed very low swelling ratios. The presence of salts, such as $\mathrm{NaCl}$, can produce a screening effect, thus lowering the repulsion between the polymer chains and, consequently, leading to a reduction of the swelling capacity. At acidic $\mathrm{pH}$, lower swelling ratios were detected, which could be attributed to the inter- and intramolecular hydrogen bonding between the acidic function groups, carboxylic and sulfonic groups. The swelling study showed high stability of the CMCg-P(SPMA)/Ag nanocomposite in different aqueous solutions, making it a suitable candidate for application in biomaterials.

\section{In vitro antibacterial and antifungal activity}

It has been reported that cellulose does not show any antibacterial activity. ${ }^{25}$ In order to impart that activity, it is necessary to incorporate some antibacterial agents, such as silver nanoparticles. As shown in Figure 7, the antibacterial and antifungal effects of the CMC-g$\mathrm{P}(\mathrm{SPMA}) / \mathrm{Ag}$ nanocomposite were evaluated by disc diffusion assays under in vitro conditions, and the average inhibition zones at different times were presented. ${ }^{17}$ Two strains (gram-negative and gram-positive) were used for performing the antimicrobial testing. After calculating the antibacterial inhibition zone, it was found that the CMC-g-P (SPMA) hydrogel did not show any bacterial activity towards any of the strains. However, CMC-g-P(SPMA)/Ag shows a relatively equal antibacterial activity towards $S$. aureus and $E$. coli (inhibition zone of $11 \mathrm{~mm}$ ). Moreover, CMC-g-P(SPMA)/Ag exhibits antifungal activity against Candida albicans, a human fungal pathogen, and Aspergillus flavus, a crop pathogen. In vitro assays revealed that the CMC-g-P(SPMA)/Ag nanocomposite was consistently more effective against $A$. flavus than against Candida albicans. The results showed that the CMC-g-P(SPMA)/Ag nanocomposite can be recommended as an effective material for protecting foodstuffs against bacterial and fungal contamination storage.

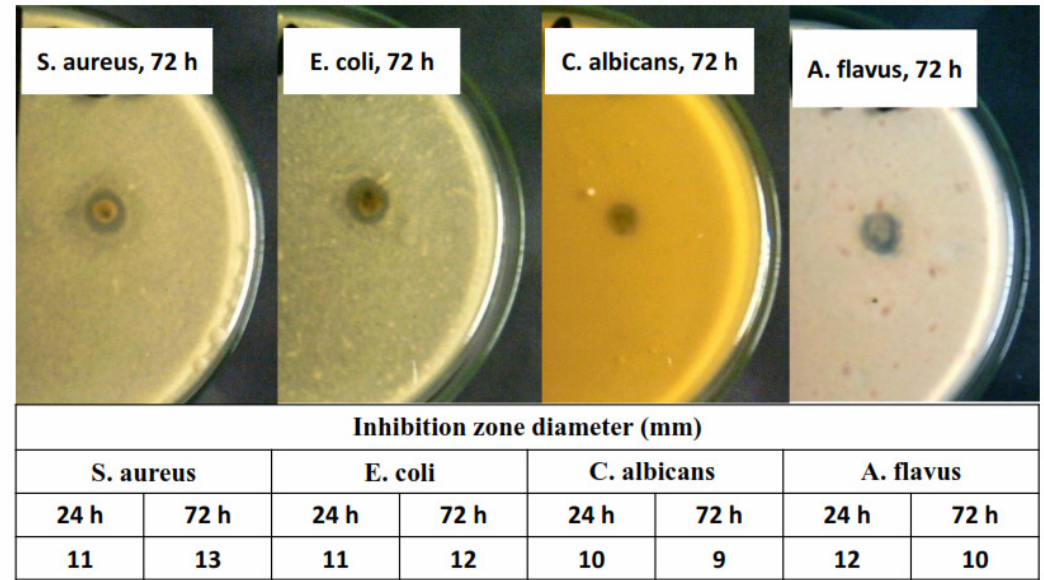

Figure 7: Antibacterial and antifungal effects of CMC-g-P (SPMA)/Ag nanocomposite after 24 and 72 hours

\section{CONCLUSION}

In summary, a carboxymethyl cellulose based hydrogel was successfully prepared as a new anionic derivative for preparing a novel CMC-g$\mathrm{P}(\mathrm{SPMA}) / \mathrm{Ag}$ nanocomposite. Silver nanoparticles with $\sim 18 \mathrm{~nm}$ diameter were well dispersed inside the network structure of the formed hydrogel. The current nanocomposite showed effective antibacterial and antifungal activities. These results suggest that the CMC-g$\mathrm{P}(\mathrm{SPMA}) / \mathrm{Ag}$ nanocomposite could be a promising antibacterial and antifungal agent for different applications in biomaterials. 
ACKNOWLEDGMENTS: The authors extend their appreciation to the Research Center for Advanced Materials (RCAMS) at King Khalid University for supporting this work through research programs under grant number RCAMS/KKU/006-19. Also, the authors acknowledge National Research Center in Egypt for supporting this work.

\section{REFERENCES}

1 A. Salama, Int. J. Biol. Macromol., 127, 606 (2019), https://doi.org/10.1016/j.ijbiomac.2019.01.130

2 M. El-Sakhawy, S. Kamel, A. Salama and H. S. Tohamy, Cellulose Chem. Technol., 52, 193 (2018), http://www.cellulosechemtechnol.ro/pdf/CCT34(2018)/p.193-200.pdf

A. Salama, J. Colloid Interface Sci., 487, 348 (2017), https://doi.org/10.1016/j.jcis.2016.10.034

4 S. Schweizer and A. Taubert, Macromol. Biosci., 7, 1085 (2007), https://doi.org/10.1002/mabi.200600283

5 A. Salama, Cellulose Chem. Technol., 52, 903 (2018),

http://www.cellulosechemtechnol.ro/pdf/CCT910(2018)/p.903-907.pdf

6 L. F. Zemljic and J. V. V. T. Kreze, Cellulose Chem. Technol., 51, $75 \quad$ (2017), http://www.cellulosechemtechnol.ro/pdf/CCT12(2017)/p.75-81.pdf

S. Dacrory, H. Abou-yousef, S. Kamel, R. E. Abou-zeid, M. Abdel-aziz et al., Cellulose Chem. Technol., $\quad 53, \quad 23 \quad$ (2019), http://www.cellulosechemtechnol.ro/pdf/CCT12(2019)/p.23-33.pdf

A. Salama, Mater. Lett., 157, 243 (2015), https://doi.org/10.1016/j.matlet.2015.05.088

M. El-sakhawy, A. Salama, S. Kamel and H.-A. Tohamy, Cellulose Chem. Technol., 52, 749 (2018), http://www.cellulosechemtechnol.ro/pdf/CCT910(2018)/p.749-757.pdf

10 A. Salama, N. Shukry and M. El-Sakhawy, Int. J. Biol. Macromol., $\quad \mathbf{7 3 ,} 72 \quad$ (2015), https://doi.org/10.1016/j.ijbiomac.2014.11.002

11 G. A. Olatunji, A. T. Kola-Mustapha, O. D. Saliu, A. B. Alabi, O. I. Abiodun et al., Korean J. Chem.
Eng., 35, 784 (2018), https://doi.org/10.1007/s11814017-0314-8

12 I. Muqbil, A. Masood, F. H. Sarkar, R. M. Mohammad and A. S. Azmi, Cancers, 3, 428 (2011), https://doi.org/10.3390/cancers3010428

13 B. Ramalingam, M. M. R. Khan, B. Mondal, A. B. Mandal and S. K. Das, ACS Sustain. Chem. Eng., 3, 2291

https://doi.org/10.1021/acssuschemeng.5b00577

(2015),

14 A. Nithya, H. L. Jeeva Kumari, K. Rokesh, K. Ruckmani, K. Jeganathan et al., J. Photochem. Photobiol. B Biol., 153, $412 \quad$ (2015), https://doi.org/10.1016/j.jphotobiol.2015.10.020

15 S. Kumari and G. S. Chauhan, Appl. Mater. Interfaces, $\quad \mathbf{6 ,} 5908$ (2014), https://doi.org/10.1021/am500820n

16 M. Ul-Islam, W. A. Khattak, M. W. Ullah, S. Khan and J. K. Park, Cellulose, 21, 433 (2014), https://doi.org/10.1007/s10570-013-0109-y

17 W. Shao, X. Liu, H. Min, G. Dong, Q. Feng et al., ACS Appl. Mater. Interfaces, 7, 6966 (2015), https://doi.org/10.1021/acsami.5b00937

18 T. Turhan, Y. A. Güvenilir and N. Sahiner, Energy, 55 , 511

(2013), https://doi.org/10.1016/j.energy.2013.01.035

19 K. A. Cavicchi, ACS Appl. Mater. Interfaces, 4, 518 (2012), https://doi.org/10.1021/am201414f

20 A. Salama and M. El-Sakhawy, Carbohyd. Polym., 113 , 500

(2014), https://doi.org/10.1016/j.carbpol.2014.07.022

21 A. Salama, R. E. Abou-Zeid, M. El-Sakhawy and A. El-Gendy, Int. J. Biol. Macromol., 74, 155 (2015), https://doi.org/10.1016/j.ijbiomac.2014.11.041

22 J. Yu, Y. Li, Q. Lu, J. Zheng, S. Yang et al., Iran. Polym. J., 25, $423 \quad$ (2016), https://doi.org/10.1007/s13726-016-0434-8

23 R. Patel, W. Seok, S. Hoon, C. Ho, H. Lee et al., Chem. Eng. J., 247, 1 (2014), https://doi.org/10.1016/j.cej.2014.02.106

24 K. Jyoti, M. Baunthiyal and A. Singh, J. Radiat. Res. Appl. Sci., 9, $217 \quad$ (2015), https://doi.org/10.1016/j.jrras.2015.10.002

25 S. Aoyagi, H. Onishi and Y. Machida, Int. J. Pharm., 330, $138 \quad$ (2007), https://doi.org/10.1016/j.ijpharm.2006.09.016 